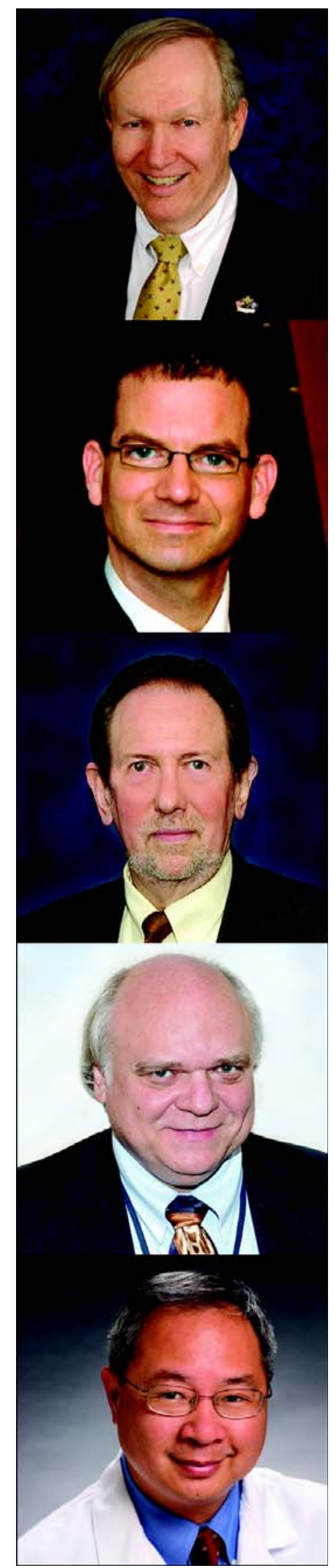

Joel Kupersmith, MD; Alex K. Ommaya, ScD; Michael E. Selzer, MD, PhD, FRCP; Robert L. Ruff, MD, PhD; Henry L. Lew, MD, PhD

\section{Traumatic brain injury research state-of-the-art conference}

The Department of Veterans Affairs (VA), treating more than 5.5 million veterans annually [1], manages the largest integrated healthcare system in the nation and is committed to providing veterans with the highest quality healthcare. Conflicts in Iraq and Afghanistan (Operation Iraqi Freedom/Operation Enduring Freedom [OIF/OEF]) have resulted in an influx of new veterans to the VA health system. From 2002 to 2008, 945,423 OIF/OEF veterans left active duty, with 42 percent $(400,304)$ obtaining VA healthcare [2]. The challenge that this trend presents to the maintenance of the VA's high standard of care is compounded by the fact that many are returning home with complex injuries, such as traumatic brain injury (TBI), frequently referred to as the signature injury of modern warfare.

Many knowledge gaps exist regarding TBI. Accordingly, in late April 2008, the VA's Office of Research and Development (ORD) sponsored a state-of-theart (SOTA) conference to advance the knowledge base in TBI. This SOTA conference identified what we know and what we need to know about TBI, from the basic science to diagnosis, evidence-based treatment, acute management, and long-term rehabilitation for TBI. The goal was to recommend for all stakeholders further research on the development of clinical practice guidelines, policies, or processes that would improve quality and outcomes of TBI care.

ORD assembled a prestigious planning committee. The planning committee developed the objectives for the SOTA conference and commissioned background articles to stimulate discussions among the 100 invited experts at the conference. Subject matter experts wrote the background articles and submitted them for peer review. Of those articles, 17 passed the rigorous review process and are published here in this special issue of Journal Rehabilitation Research and Development (JRRD). The articles cover various aspects of TBI and its comorbidities. Topics include-

1. Acute clinical care for TBI: Continuation of care from the battlefield to the Department of Defense (DOD) and the VA.

2. Pathology of blast-related brain injury.

3. Infectious complications in OIF/OEF veterans with TBI.

4. Posttraumatic epilepsy.

5. Prevalence of chronic pain, posttraumatic stress disorder (PTSD), and persistent postconcussive symptoms in OIF/OEF veterans: The polytrauma clinical triad.

6. Treating veterans with comorbid mild TBI (mTBI) and PTSD.

7. Advances in neuroimaging of TBI and PTSD.

8. Approaches to war-related mild to moderate TBI. 
9. Auditory dysfunction associated with TBI.

10. Visual function in TBI.

11. Impact of dual sensory loss on functional rehabilitation.

12. Sleep disorders in TBI.

13. Sleep apnea in TBI.

14. Pharmacology in TBI.

15. Families of patients with polytrauma.

16. Comorbidity factors and vocational outcomes for veterans with TBI.

17. Community integration for individuals with TBI. In addition, an important milestone in enhancing evidence-based practice of TBI is the publication of the VA/DOD mTBI Clinical Practice Guideline in this special issue of $J R R D$. We wish to acknowledge all clinicians, researchers, administrators, and JRRD staff who contributed to the success of this special issue. This collaboration represents a small but significant step in VA's efforts to understand TBI, with the ultimate goal to deliver the best possible care to the veterans we serve.

\section{REFERENCES}

1. VA Benefits \& Health Care Utilization [Internet]. Washington (DC): Department of Veterans Affairs; [updated 2009 Apr 17]. Available from:

http://www1.va.gov/vetdata/docs/ 4X6_spring09_sharepoint.pdf/.

2. VHA Office of Public Health and Environmental Hazards. Analysis of VA health care utilization among US Global War on Terrorism (GWOT) veterans. Washington (DC): Veterans Health Administration; 2009.

Joel Kupersmith, MD; ${ }^{1}$ Alex K. Ommaya, ScD; ${ }^{1}$ Michael E. Selzer, MD, PhD, FRCP; ${ }^{2}$ Robert $L$. Ruff, MD, PhD; ${ }^{1}$ Henry L. Lew, MD, PhD ${ }^{3^{*}}$

${ }^{1}$ VA, ORD, Washington, DC; ${ }^{2}$ Rehabilitation Research \& Development Service, Veterans Health Administration, Washington, DC; ${ }^{3}$ VA Palo Alto Health Care System, Palo Alto, CA, and VA Boston Healthcare System, Physical Medicine and Rehabilitation, Boston, MA

*Email: henry.lew@va.gov

DOI:10.1682/JRRD.2009.06.0085 